\title{
A Hybrid Approach for COVID-19 Detection Using Biogeography-Based Optimization and Deep Learning
}

\author{
K. Venkatachalam ${ }^{1}$, Siuly Siuly ${ }^{2}$, M. Vinoth Kumar ${ }^{3}$, Praveen Lalwani ${ }^{1}$, Manas Kumar Mishra ${ }^{1}$ and \\ Enamul Kabir ${ }^{4}$,
}

\footnotetext{
${ }^{1}$ Department of Computer Science and Engineering, CHRIST (Deemed to be University), Bangalore, 560074, India

${ }^{2}$ Institute for Sustainable Industries \& Liveable Cities, Victoria University, Melbourne, 14428, Australia

${ }^{3}$ Department of Computer Science and Engineering, Anna University, University College of Engineering, Dindigul, 624622, India

${ }^{4}$ School of Sciences, University of Southern Queensland, Toowoomba, Darling Heights, 4350, Australia

${ }^{*}$ Corresponding Author: Enamul Kabir. Email: Enamul.Kabir@usq.edu.au

Received: 10 March 2021; Accepted: 19 April 2021
}

\begin{abstract}
The COVID-19 pandemic has created a major challenge for countries all over the world and has placed tremendous pressure on their public health care services. An early diagnosis of COVID-19 may reduce the impact of the coronavirus. To achieve this objective, modern computation methods, such as deep learning, may be applied. In this study, a computational model involving deep learning and biogeography-based optimization (BBO) for early detection and management of COVID-19 is introduced. Specifically, BBO is used for the layer selection process in the proposed convolutional neural network (CNN). The computational model accepts images, such as CT scans, $\mathrm{X}$-rays, positron emission tomography, lung ultrasound, and magnetic resonance imaging, as inputs. In the comparative analysis, the proposed deep learning model CNN is compared with other existing models, namely, VGG16, InceptionV3, ResNet50, and MobileNet. In the fitness function formation, classification accuracy is considered to enhance the prediction capability of the proposed model. Experimental results demonstrate that the proposed model outperforms InceptionV3 and ResNet50.
\end{abstract}

Keywords: Covid-19; biogeography-based optimization; deep learning; convolutional neural network; computer vision

\section{Introduction}

The outbreak of COVID-19 [1], also known as coronavirus caused by SARS-CoV-2, has become a public health hazard for the international community. This outbreak was announced by the WHO as a public health emergency of international concern on January 30, 2020 and was officially declared as a pandemic [2-4] on March 11, 2020. COVID-19 is a contagious disease that can be transmitted among humans. The number of confirmed cases worldwide has risen exponentially over the past year, reaching 125,436,255 (with 101,304,833 recoveries and 2,756,767 deaths) as of March 25, 2021.

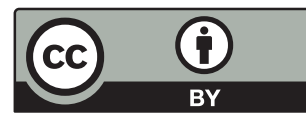

This work is licensed under a Creative Commons Attribution 4.0 International License, which permits unrestricted use, distribution, and reproduction in any medium, provided the original work is properly cited. 
The mass transportation and large gatherings during the Chinese festivals last year resulted in the rapid spread of the virus in other parts of China [5]. The amount of deaths attributable to this virus has already exceeded the numbers reported in other pandemics, such as SARS and MERS [6]. As reported in [7-9], the usual manifestations of COVID-19 include fever, respiratory symptoms, pneumonia, and low white blood cell count [10,11]. The virus has also been described as an acute respiratory tract syndrome [12]. Its early symptoms include difficulty in breathing and lung disease accompanied by hyperthermia. The aberration seen in lungs due to viruses other than COVID-19 is visually similar or peripheral to that reported in viral pneumonia and other bacterial pathogens [13]. To prepare countries in handling the pandemic and make efficient decisions, viral activity predictive modelling studies are performed by using big data. Some of the issues being faced by various countries due to COVID-19 include the following:

1. early and accurate diagnosis of the disease;

2. spreading awareness;

3. delivering relevant guidelines;

4. preventing the proliferation of hoaxes on social media;

5. saving health care experts some time from doing laborious jobs; and

6. ensuring adherence to social distancing norms.

Machine learning can play a critical role in solving these issues [14-17]. Current medical informatics and experience imply that reverse transcription polymerase chain reaction (RT-PCR) [18] tests used for the detection or identification of viral RNA has low sensitivity especially at the initial stage $[19,20]$. This problem has also been reported in laboratory tests [21] and can be ascribed to many factors, including sample preparation and quality control [22].

\subsection{Key Findings for the Early Diagnosis of COVID-19}

In the identification process, an inflammation of air sacs in the lungs is detected in the form of pneumonia, and tracking the bilateral lung involvement can help identify various stages of the disease, such as initial, intermediate, and last stage. Easily available imaging equipment, such as chest X-rays and thoracic CT, greatly facilitates the diagnosis of such aberrations [23,24]. Therefore, a large number of abnormal chest CT images from COVID-19 patients have been gathered and examined [25,26]. From this pool of data, bilateral pulmonary grounded glass shadow [27] and nodules were observed, which indicate severe levels of the disease. Therefore, in the early diagnosis of COVID-19 and community-acquired pneumonia [28,29], characterizing the relationship among multiple features from CT images introduces the possibility for an automated diagnosis of the disease. Sample chest CT images are shown in Figs. 1a-1c and 2.

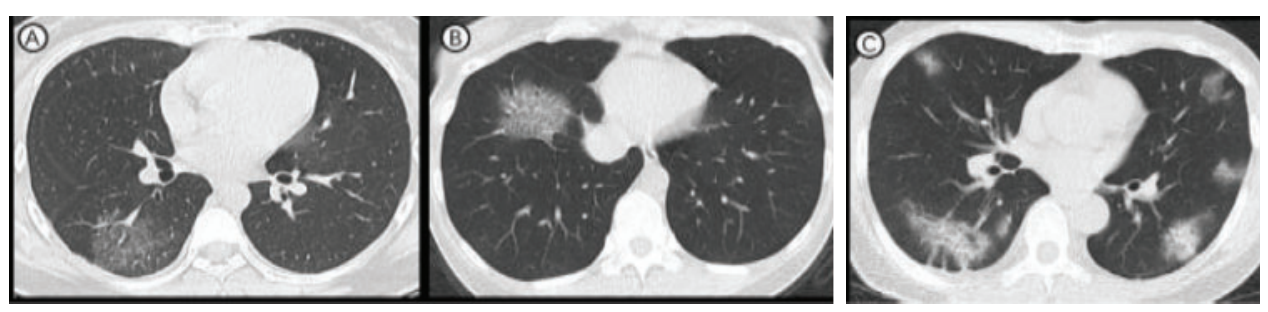

(a)

(b)

(c)

Figure 1: CT images of COVID-19 patients 

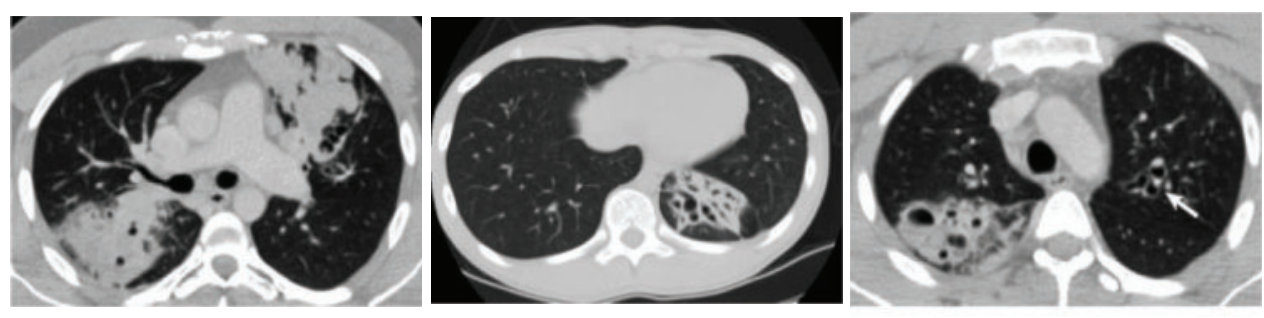

Figure 2: CT images of patients with community-acquired pneumonia

\subsection{Research Problem and Objectives}

COVID-19 detection via imaging has become a leading research area. However, the existing approaches in this domain [30] have low prediction accuracy due to the improper selection of layers. Instead of randomly selecting layers in a convolutional neural network (CNN), a customized selection by using optimization techniques that enhance prediction accuracy can be used.

This research aims to propose a new method for the early prediction of COVID-19 to cease further transmission and save more lives. Various images, such as X-rays, are being analyzed in the prediction process by using the deep learning model. However, a pre-trained model may be considered in hardware development, which results in low accuracy. Therefore, the classification accuracy of real-time devices needs to be enhanced. To achieve this objective, a new model is developed via biography-based optimization (BBO).

\subsection{Research Contributions}

The highlights of this article are as follows:

- This article extensively reviews the most important technologies that use computational methods for COVID-19 detection.

- Most of the solutions provided by researchers are based on computed tomography images. Therefore, this article also categorizes these solutions during the analysis.

- We propose a novel CNN for classifying COVID-19 positive patients based on chest X-rays.

- $\mathrm{BBO}$ is used for the layer selection process in $\mathrm{CNN}$.

- Classification accuracy is taken into consideration in the fitness function composition.

- The proposed model is compared with existing models to demonstrate its superiority.

\subsection{Thesis Organization}

The rest of this article is organized as follows. Section 2 describes the recent advances in COVID-19 diagnosis based on image data. Section 3 presents an overview of BBO. Section 4 presents the proposed model for classifying COVID-19 positive images. Section 5 presents an experimental and comparative analysis of the existing models. Section 6 concludes the paper and presents directions for future research.

\section{Related Work on COVID-19 Prediction}

This section presents the recent applications of machine learning in COVID-19 diagnosis. These existing applications are categorized into CT-scan- and X-rays-image-based approaches. The advantages of the proposed model over extant ones are also highlighted. 


\subsection{CT-Scan-Based Approaches}

Chest CT images of patients are classified into three groups, namely, COVID-19, influenzaA viral pneumonia, and healthy cases. Researchers in [30] developed a dual sampling attention network for diagnosing COVID-19 from community-acquired pneumonia cases by using chest CT. They used a 3D CNN to determine the infection regions in lungs, but their proposed model reported an accuracy of only $87 \%$, which can be further improved by using other computational models. Researchers in [31] attempted to identify pulmonary vein arteries from chest CT images by using 3D CNN. While their proposed model obtained a maximum accuracy of near 93\%, this level can be improved further by using other computer vision approaches. Specifically, they extracted the features of interstitial lung diseases and used deep CNN and transfer learning afterward. However, this process reduced the accuracy of their model to $91 \%$, which needs to be improved further before their model can be adopted in real-world applications. Researchers in [32] proposed a supervised dis-convolutional framework with an aim to detect pulmonary fissure in CT. However, their proposed fissure methods are limited to handcrafted and local features, thereby leading to low specificity. These methods obtained an accuracy of $98 \%$. Moreover, they attempted to classify pulmonary nodules from CT images by using a multi-view convolution network and achieved a maximum accuracy of around 94\%. However, to achieve further improvements, other computational models can be adopted.

In [33], 3D CNN was used to identify pulmonary nodules from chest CT images, and the experimental results were compared in terms of their accuracy. A maximum accuracy of $95 \%$ was obtained. Researchers in [34] predicted the severity of tuberculosis from CT pulmonary images by using a 3D block-based residual deep learning network. They reported an accuracy of $92.7 \%$, which can be further improved by altering the depth-ResNet parameters. Researchers in [35] used a deep transfer learning technique to classify patients infected with COVID-19. Similar work was done by other researchers who reported a $96.2 \%$ accuracy. These researchers also proposed a deep-learning-based system for segmenting and quantifying infected regions in lungs based on chest CT. They also applied a VB-Net neural network for image segmentation and obtained a dice similarity index of $91.6 \%$. However, the benchmark was not reached in real time. Therefore, another computation method with fine tuning parameters should be adopted. Other researchers proposed efficient feature extraction techniques, such as ResNet and deep learning models, and obtained significant results. Researchers in [36] proposed a deep learning framework known as COVIDX-Net, whose structure is based on seven different deep CNNs, namely, VGG19, DenseNet201, InceptionV3, ResNetV2, InceptionResNetV2, Exception, and MobileNetV2. Those CNNs that outperformed others include VGG19 and DenseNet201, which reported an accuracy of $90 \%$. However, combining analytical models with CNNs can lead to further improvements. Researchers in [37] proposed a novel technique with the objective of identifying lung abnormalities or aberrations based on the deep learning framework and reported an accuracy of around 97.7\%.

\subsection{X-ray-Based Approaches}

X-rays are considered acceptable alternatives to chest CT scans given its greater sensitivity to pulmonary diseases compared with the latter. One major reason for switching from chest CT images is their sensitivity to pulmonary diseases and limited portability seeing that these images require more staff and an additional hour to process, which leads to the possibility of infecting hospital staff. By contrast, chest X-rays can be captured in a highly isolated environment and requires less time to process. Sample chest X-rays of infected and non-infected patients are shown in Fig. 3. 


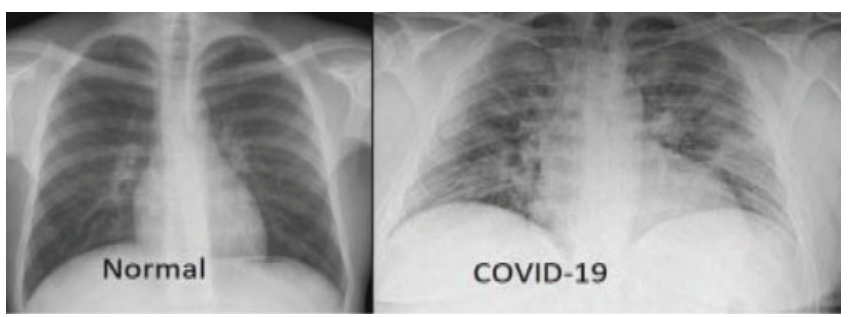

Figure 3: X-ray images of non-COVID-19 (left) and COVID-19 patients (right)

Previous studies have utilized the power of deep learning algorithms and trained neural networks to detect the frontal view of chest radiographs for 14 pathologies (CheXNeXt, 2018). They achieved an accuracy ranging from $80 \%$ to $85 \%$, which is too low to be considered for actual practice.

Researchers in [38] demonstrated the use of an iteratively pruned deep learning model for detecting the pulmonary manifestation of COVID-19 based on chest X-rays. They compared the performance of customized CNN with that of ImageNet-pertained models, namely, VGG16, VGG19, InceptionV3, Xception, InceptionResNetV2, MobileNetV2, DenseNet-201, and NasNetmobile. The customized CNN outperformed the other tested models with a maximum accuracy of $99 \%$.

Researchers in [39] proposed a hybrid COVID-19 detection model that extracts small regions from chest X-rays that contain identifying features of COVID-19 based on the improved marine predators algorithm (IMPA). They also applied the ranking-based diversity reduction strategy to enhance the performance of IMPA in a limited number of iterations. In the experimental analysis, deep learning algorithms (i.e., $\mathrm{CNN}$ ) have been proven to be strongly optimistic for image classification, but they require more data for testing and classification to achieve an improved accuracy. However, during the pandemic, obtaining a sufficient number of chest radiographs for training the algorithm with higher precision is difficult.

In this article, we utilized three CNNs for diagnosing COVID-19, namely, ResNet50, InceptionV3, and Inception-ResNetV2 [40]. ResNet50 obtained the highest accuracy of 97\%, but a more rigorous testing of models should be implemented.

\subsection{Advantage of the Proposed Model Over Existing Methods}

The merits of the proposed model are as follows:

- In the proposed work, a novel approach for layer selection based on BBO is devised. This method uses classification accuracy as a fitness function in contrast to existing works where the classification accuracy is low due to improper layer selection.

- Some researchers attempted to capture the solution by using metaheuristics, such as GA, but BBO obtains a better solution with a smaller number of iterations [41].

- Deep transfer learning is used to train the CNN classification model with VGG16 and InceptionV3, thereby reducing pre-processing and feature selection works in contrast to existing techniques.

- The proposed technique obtained a classification accuracy of $97.98 \%$ for publicly available COVID-19 datasets, and this accuracy is comparatively better than that of other techniques. 


\section{Overview of Biogeography-Based Optimization}

Introduced by Simon et al. in 2008 [41], BBO distributes biological species in a geographical way. To represent a geographical zone, an index called habitat suitability index (HSI) is considered. Another index called suitability index variable (SIV) is used to represent the area and livelihood conditions. The goodness of each habitat is represented by the HSI value and the number of species. The HSI value can be increased by adopting features from the high HSI solution, and this mechanism is called BBO.

Fig. 4 illustrates the species abundance process wherein the immigration and emigration rates are represented by $\lambda$ and $\mu$, respectively. In the immigration curve, the immigration rate is estimated to be maximum (I) when the habitat consists of zero species. Fig. 4 also shows that $\lambda$ decreases along with increasing species and reaches zero when the habitat has the maximum number of species. Fig. 4 also shows that the emigration rate increases along with the number of species and reaches 0 when no species are in the habitat. In the balanced condition, both rates are equal as indicated by (So).

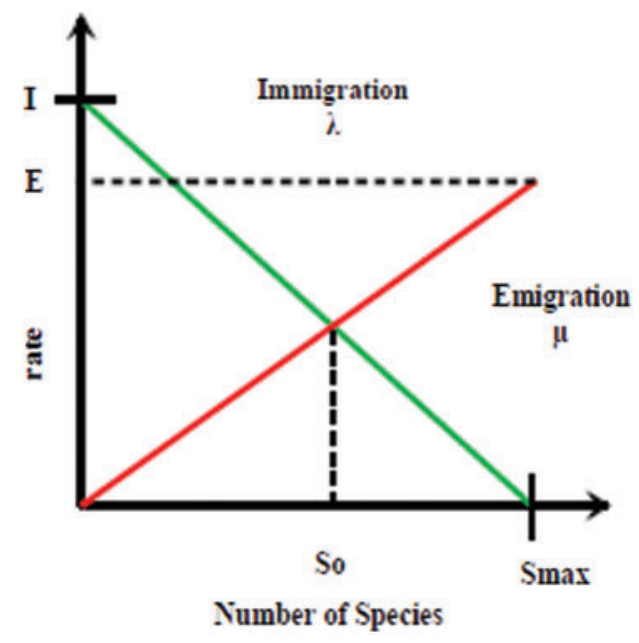

Figure 4: Species representation of a single habitat follows:

The immigration and emigration rates are formulated from the straight-line curve (Fig. 4) as

$\mu=\frac{E \times n_{k}}{n_{t}}, \quad \lambda=I\left(1-\frac{n_{k}}{n_{t}}\right)$

where $n k$ represents the number of species inside the habitat, and $n t$ denotes the total number of species.

The $\mathrm{BBO}$ mechanism is based on migration and mutation processes as described below.

\subsection{Migration}

Let represent the optimization problem. The population members represent the candidate solution, with each member having an $\mathrm{n}$ dimension vector called habitat and being represented by SIV. The fitness value of a habitat is estimated by using the number of species and HSI value. In the solution improvement process, a low HSI solution shares the same features as the high HSI 
solution vector based on $\lambda$ and $\mu$. One habitat (Hi) is initially selected by using $\lambda$, and another habitat $(\mathrm{Hj})$ is selected based on $\mu$. In the migration process, some $\mathrm{SIVs}$ from $\mathrm{Hj}$ are transferred to $\mathrm{Hi}$.

\subsection{Mutation}

The mutation process in BBO is reflective of a natural disaster. Specifically, similar to disasters, the mutation has an unpredictable effect when applied on a habitat. This effect depends on the number of species and is estimated by using Eq. (1). In this process, the probability is estimated for each habitat. A high probability corresponds to low chances of mutation, that is, the solution is not optimal, and vice-versa.

$P_{n k}{ }^{h}= \begin{cases}-\left(\boldsymbol{\lambda}_{\mathbf{n k}}+\boldsymbol{\mu}_{\mathbf{n k}}\right) P_{n k}+\boldsymbol{\mu}_{\mathbf{n k}+1} P_{n k+1}, & n k=0 \\ -\left(\boldsymbol{\lambda}_{\mathbf{n k}}+\boldsymbol{\mu}_{\mathbf{n k}}\right) P_{n k}+\boldsymbol{\mu}_{\mathbf{n k}+1} P_{n k+1}+\boldsymbol{\mu}_{\mathbf{n k}-1} P_{n k-1,}, & 1 \leq n k \leq S_{\text {max }}-1 \\ -\left(\boldsymbol{\lambda}_{\mathbf{n k}}+\boldsymbol{\mu}_{\mathbf{n k}}\right) P_{n k}+\boldsymbol{\mu}_{\mathbf{n k}-1} P_{n k-1}, & n k=S \_ \text {max }\end{cases}$

where $m(n k)$ represents the mutation rate of $n k$ species, $\mathrm{m}_{\max }$ is the maximum mutation rate, and Pmax represents the maximum mutation probability. This mutation has three key advantages. First, this process has a greater variety of candidate solutions. Second, resist solution to disrupt having HSI value. Third, this process improves both the high and low HSI solutions. However, the mutation process may degrade the solution vector.

\section{Proposed Approach: BBO-Based Deep Learning Model}

This section presents the notations and abbreviations, an overview of the proposed model, a description of habitat representation and initialization, the fitness function, the migration and mutation processes, the pseudo code of the BBO-based deep learning model, and the layers of the optimized model.

\subsection{Overview of the Proposed Model}

As shown in Fig. 5, in the initial step, all layers of each type in the CNN are randomly initialized, and the solution vectors are framed. Afterward, the classification accuracy of all random solution vectors (habitats) is estimated as shown in Fig. 5b. Thereafter, the habitat (i.e., solution vector) is updated with the help of migration and mutation processes by using the immigration and emigration rates. The $\mathrm{BBO}$ mechanism is repeated, that is, the migration and mutation steps are repeated until the best classification accuracy is achieved as shown in Fig. 5a.

\subsection{Habitat Representation and Initialization}

In this process, the habitat represents the type of layers in CNN and is initialized with a number of layers of each type. Let Hi represent the habitat (i.e., type of layers), where $\mathrm{H}_{\mathrm{i}}=\left\{\mathrm{H}_{1}\right.$, $\left.\mathrm{H}_{2}, \ldots, \mathrm{H}_{\mathrm{t}}\right\}$. Each SIV is randomly initialized between 1 and $\mathrm{L}_{\max }$, where $\mathrm{L}_{\max }$ represents the maximum number of layers of each type that can be considered.

\subsection{Fitness Function Formulation}

Classification accuracy is considered in the fitness formulation process as shown in Eq. (4). Increasing the classification accuracy also increases the fitness score. Therefore, the objective is to maximize Eq. (4). In $\mathrm{BBO}$, the fitness score represents the goodness of habitat as reflected by the HSI value and number of species. 


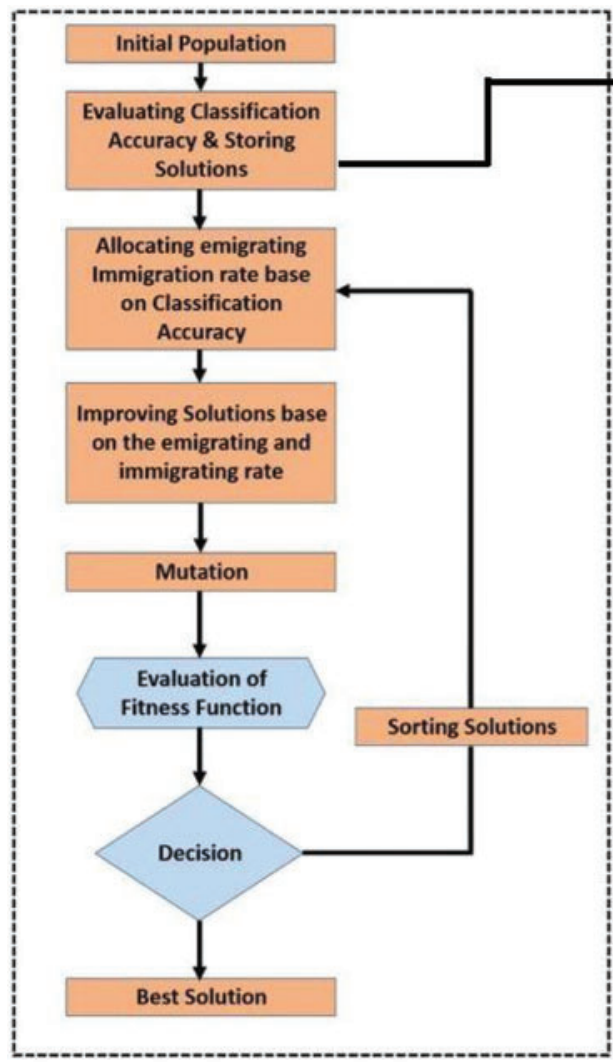

(A)

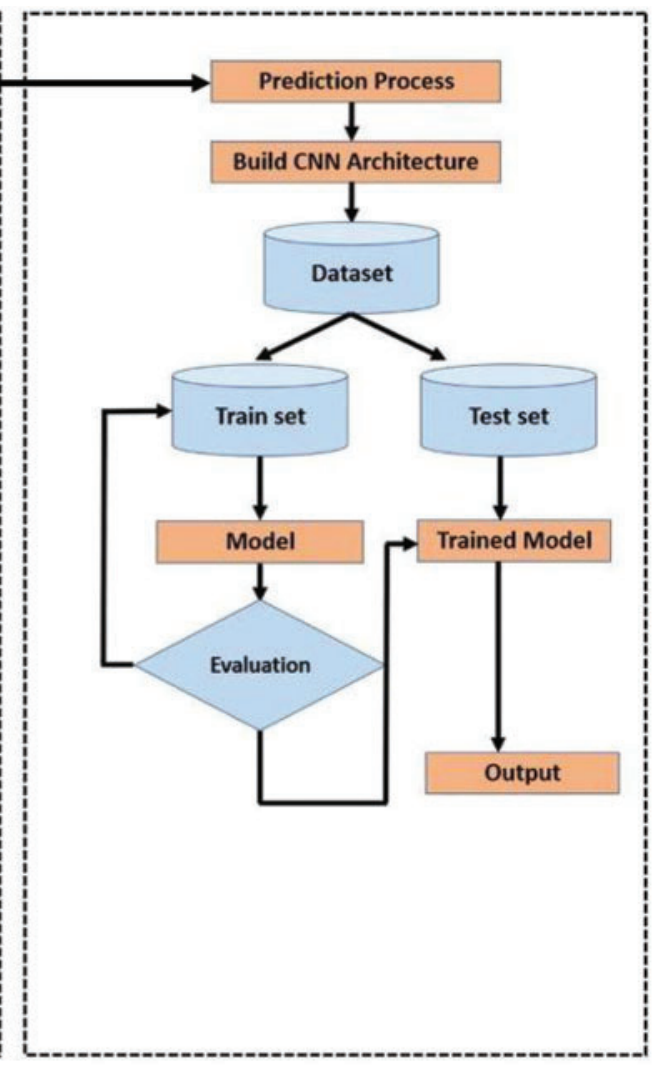

(B)

Figure 5: Optimized deep learning model

\subsection{Migration Process}

The migration process is divided into two steps. In the first step, two habitats are selected probabilistically. The first habitat $\left(\mathrm{H}_{\mathrm{i}}\right)$ is chosen probabilistically based on immigration rate $\left(\lambda_{\mathrm{i}}\right)$. Meanwhile, the second habitat $\left(\mathrm{H}_{\mathrm{j}}\right)$ is selected probabilistically based on emigration rate $\left(\mu_{\mathrm{j}}\right)$. In the second step, the SIVs from habitat $\mathrm{H}_{\mathrm{j}}$ are transferred to $\mathrm{H}_{\mathrm{i}}$, that is, a number of layers of each type appears from the high HSI solution to the low HSI one. One position (i.e., $\mathrm{p}_{\mathrm{i}}$ ) is then randomly generated between the s1 and $\mathrm{t}^{\text {th }}$ dimensions and, from position $p_{i}$ to the last position, all SIVs are transferred from $\mathrm{H}_{\mathrm{j}}$ to $\mathrm{H}_{\mathrm{i}}$.

\subsection{Mutation Process}

In the mutation process, we consider the number of layers ranging from 1 to 25 of each type. In this mechanism, a habitat is selected while considering mutation probability. Afterward, one SIV is selected between the 1 to $t^{\text {th }}$ dimensions randomly and replaced with a random selection of layers. 


\subsection{Step-Wise Approach of the Proposed Framework}

The proposed model involves several steps, namely, BBO, classification accuracy and solution evaluation, and prediction process initiation. The model workflow is illustrated in Fig. 5, and its algorithm is shown in Algorithm 1.

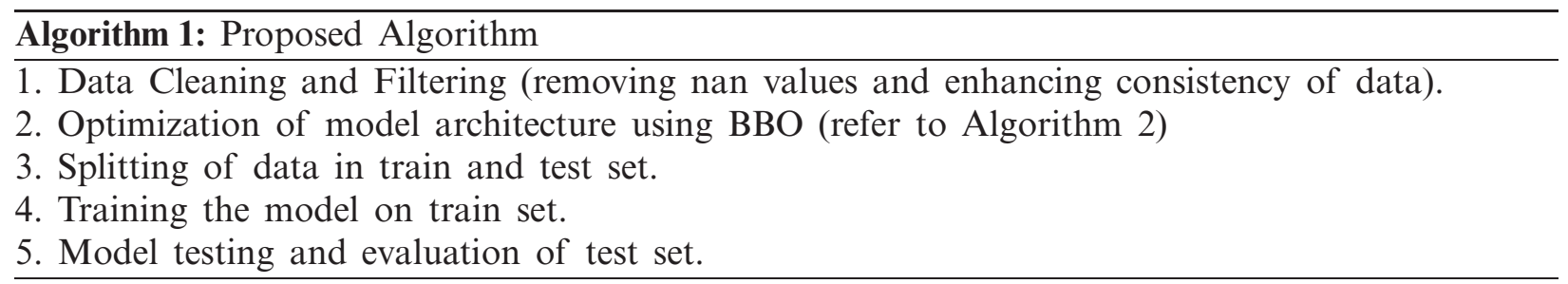

\subsubsection{Workflow}

The workflow is divided into three phases after BBO (i.e., after the best solution for the prediction initiation is evaluated). BBO is briefly discussed and illustrated above.

The three phases include training the model on the train set, testing the model on the test set, and evaluating the model on the test set. The evaluation metrics are briefly discussed in Section 6.

\subsubsection{Layer Description of the Obtained Optimized Deep Learning Model}

The obtained optimized CNN comprises 2 conv2D layers, 2 maxpool layers, 1 flatten layer, and 1 dense layer. The first conv2D layer consists of 32 filters, whereas the other conv2D layer consists of 64 filters. Chest X-rays with dimensions of $(244,244)$ are pre-processed (inspired from VGG16 image pre-processing). The proposed model is illustrated in Fig. 6. The dataset is split into three sets, namely, the train set, test set, and validation set. The model is trained for 50 epochs and is evaluated based on its classification accuracy. Section 5 presents a detailed description of the results.

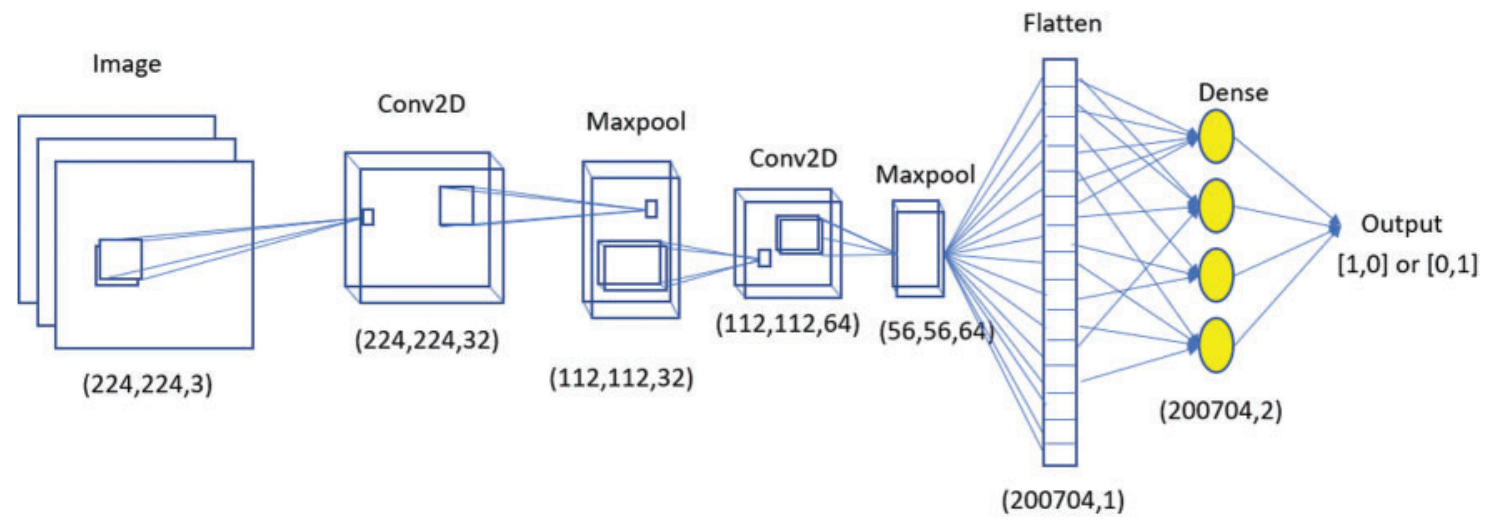

Figure 6: Optimized CNN model architecture 


\subsection{Algorithm of the BBO-Based Deep Learning Model}

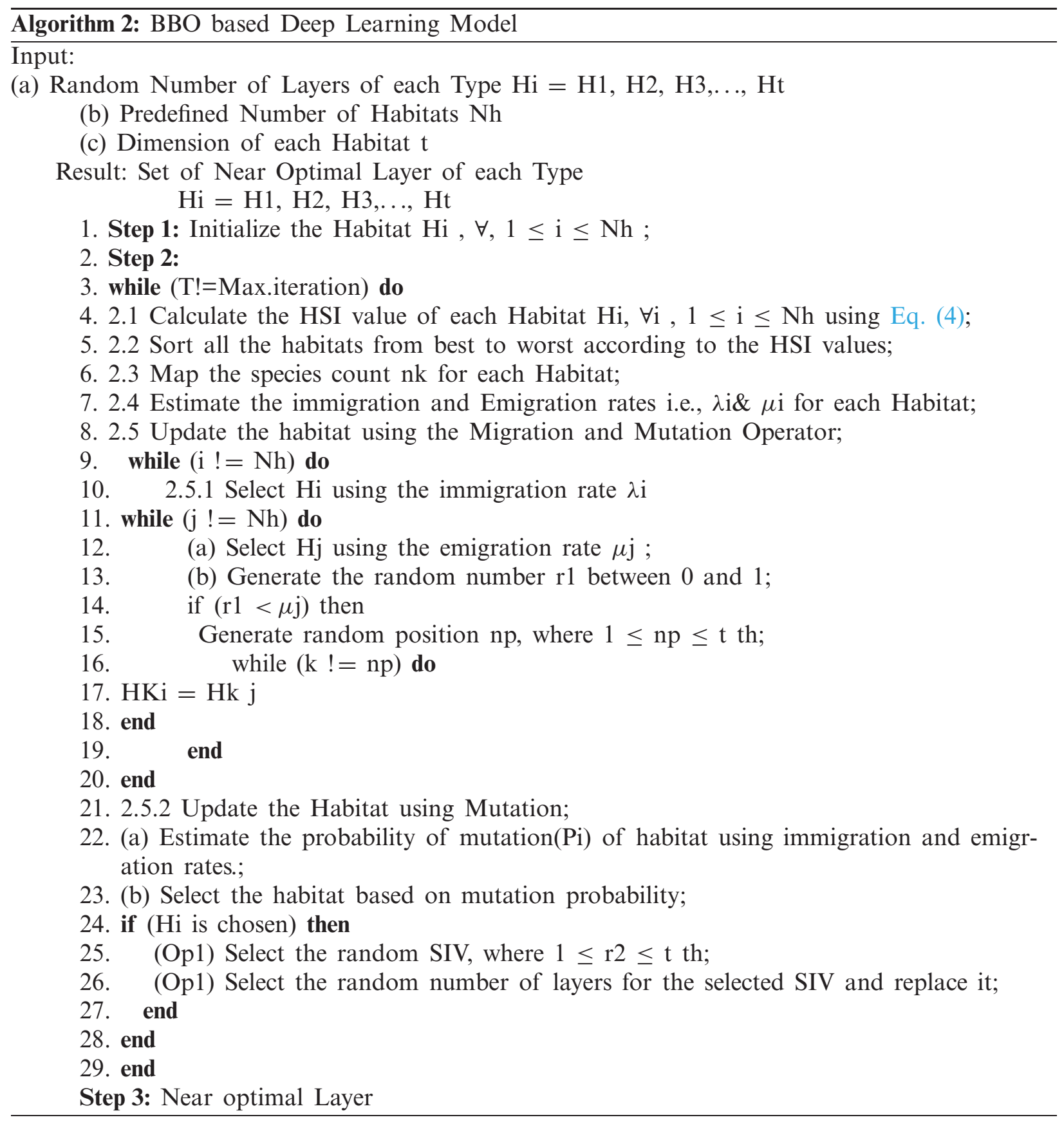




\section{Experimental Analysis}

The proposed model is implemented along with other models, namely, VGG16, MobileNet, InceptionV3, and ResNet50, on a public dataset [35]. Images are taken from various collections of datasets [35]. A total of 413 COVID-19 positive images and 439 normal pneumonia-infected images are obtained. The proposed model reaches BBO convergence after 200 iterations.

\subsection{Classification Accuracy}

Classification accuracy is computed as the ratio of correctly classified samples with respect to the total number of samples and is represented by $\mathrm{C}(\mathrm{A})$.

$$
C(A)=\frac{S_{c}}{S_{t}}
$$

where $S_{c}$ indicates the correctly classified samples, and $S_{t}$ denotes the total number of samples. For binary classification problems, accuracy as either positive or negative values as shown in Eq. (4).

$$
C(A)=\frac{T_{p}+T_{n}}{T_{p}+T_{n}+F_{p}+F_{n}}
$$

where $T_{p}, T_{n}, F_{p}$, and $F_{n}$ represent true positive, true negative, false positive, and false negative, respectively.

The metrics of the confusion matrix are mathematically represented in Eqs. (5)-(7).

Accuracy $=\frac{T_{p}+T_{n}}{T_{p}+T_{n}+F_{p}+F_{n}}$

Sensitivity $=\frac{T_{p}}{T_{p}+F_{n}}$

Specificity $=\frac{T_{n}}{T_{n}+F_{p}}$

\subsection{Model Training Phase Evaluation}

The model training phase is evaluated based on training and validation losses. The results are illustrated in Figs. 7a-7e. Training loss denotes the error on the training set in each epoch, whereas the validation loss denotes the error on the validation set (which is $20 \%$ of the training set).

As shown in Fig. 7, InceptionV3 has a monotonically decreasing loss curve with Li (Initial Loss) $=0.45$ and Fi (Final Loss) $=0.1$. The proposed CNN also has a significant decreasing loss curve with $\mathrm{Li}=4.6$ and $\mathrm{Fi}=0.2$. ResNet50 has a loss curve with $\mathrm{Li}=0.24$ and $\mathrm{Fi}=0.21$, whereas MobileNet has a loss curve with $\mathrm{Li}=0.069$ and $\mathrm{Fi}=0.01$. In VGG16, the curve begins with loss $(\mathrm{Li}=0.14)$ and ends with another loss $(\mathrm{Fi}=0.02)$. These results indicate that VGG16 and the proposed CNN model have the best fit among all models during the training phase. 


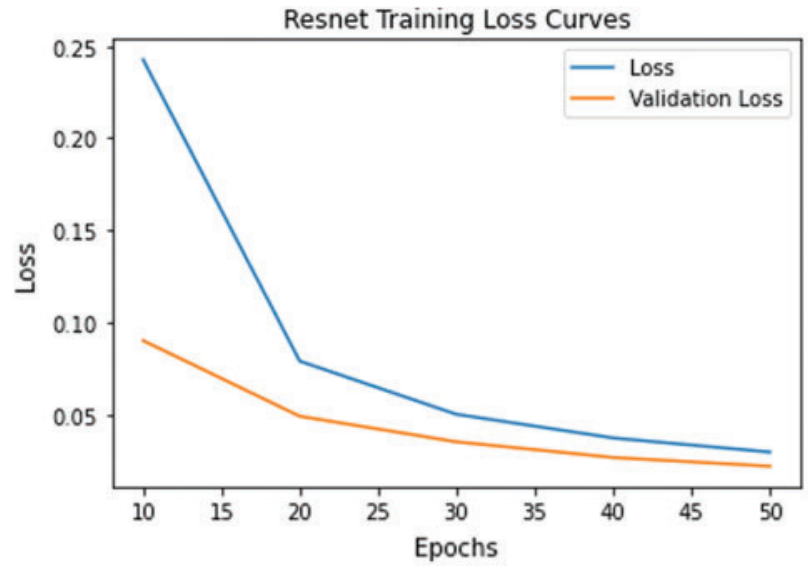

(a)

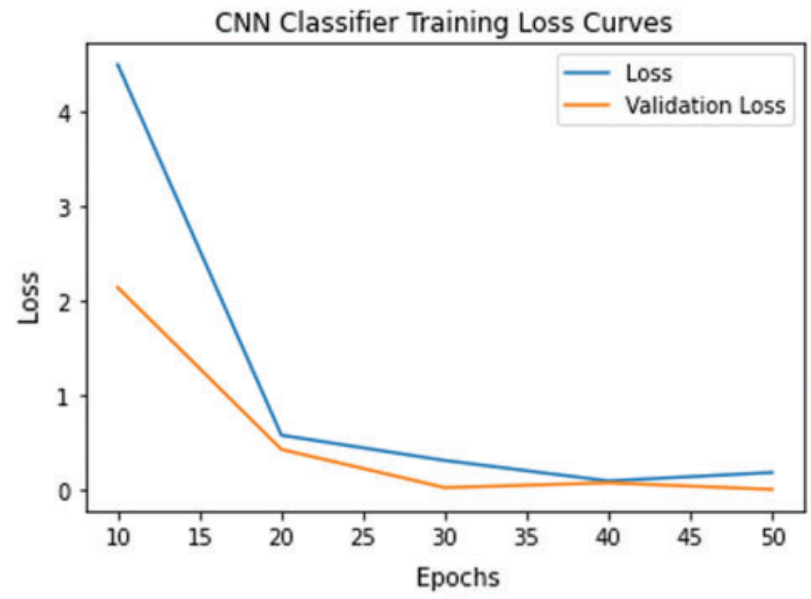

(c)

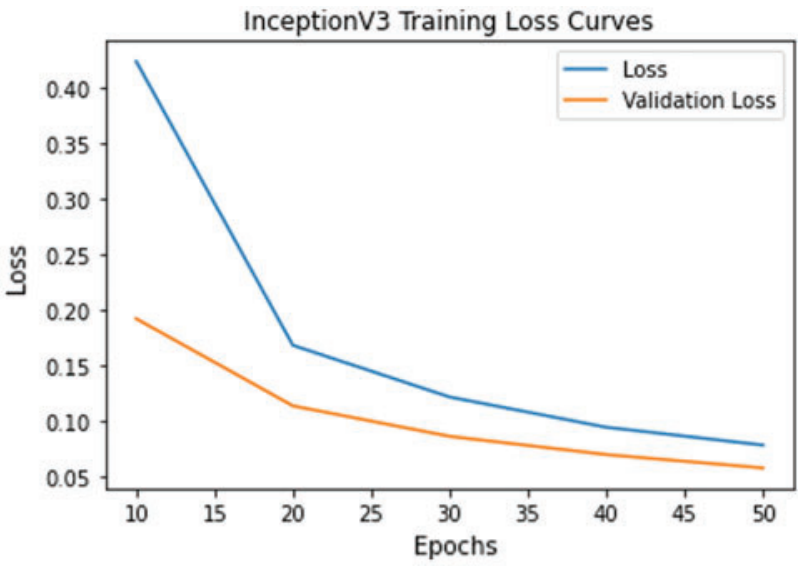

(b)

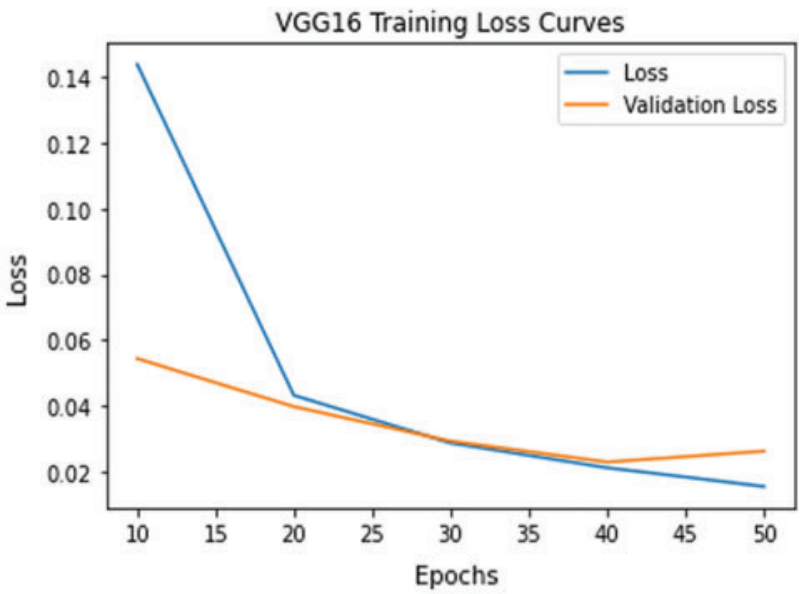

(d)

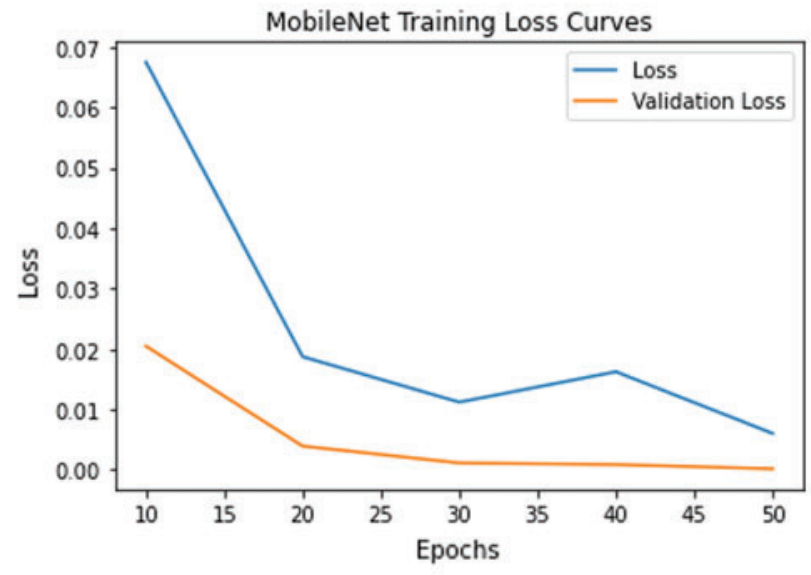

(e)

Figure 7: Loss Information patterns of (a) ResNet50, (b) InceptionV3, (c) CNN, (d) VGG16, and (e) MobileNet 


\subsection{Experimental Results}

The results obtained from the confusion matrix are shown in Tab. 1 and illustrated in Figs. 8a-8d.

Table 1: Comparative analysis of machine learning models

\begin{tabular}{lllll}
\hline Models & Test accuracy & Train accuracy & Specificity & Sensitivity \\
\hline Proposed CNN & 98.5 & 97.6 & 100 & 97 \\
VGG16 & 99.01 & 98.2 & 100 & 98.01 \\
InceptionV3 & 97 & 97.2 & 97.01 & 98 \\
ResNet50 & 100 & 96.8 & 100 & 100 \\
MobileNet & 100 & 99.1 & 100 & 100 \\
\hline
\end{tabular}

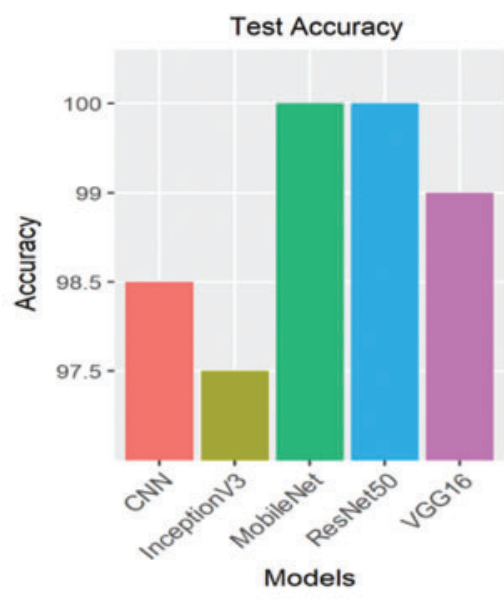

(a)

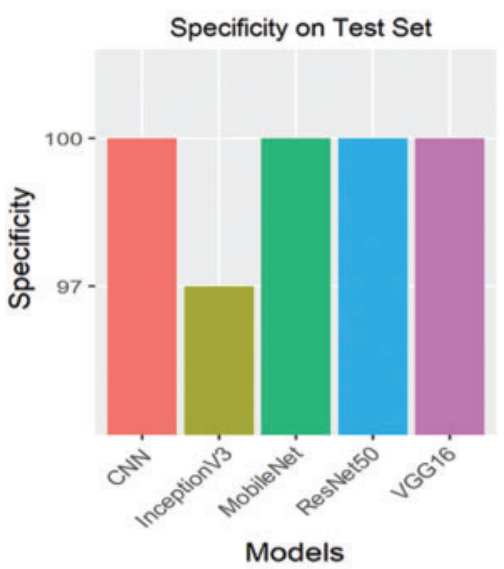

(c)

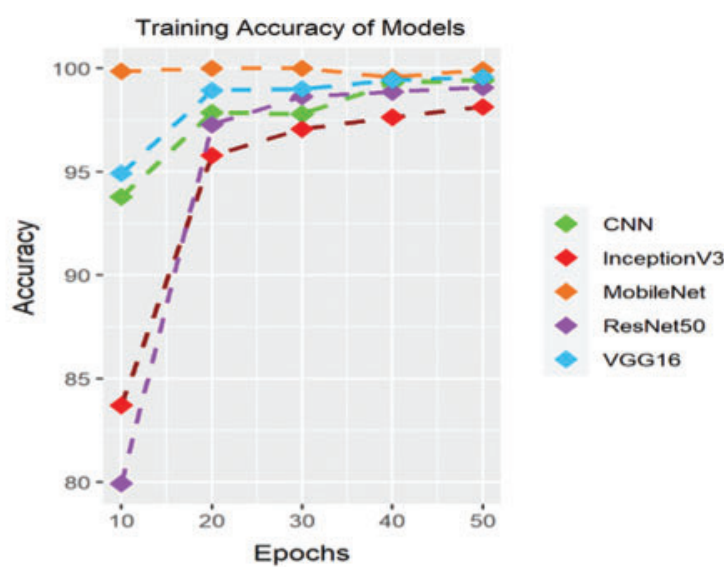

(b)

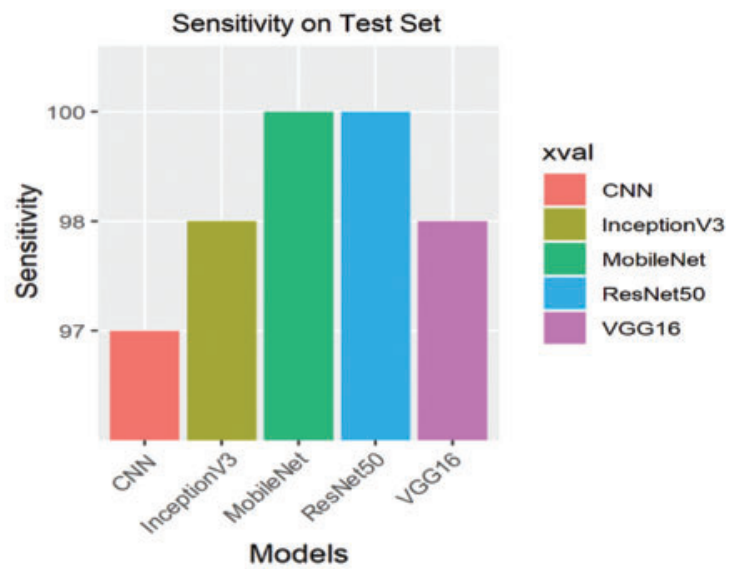

(d)

Figure 8: $(a-b)$ Accuracy on the train and test sets, and (c-d) specificity and sensitivity on the test set 
A. Training Accuracy: In the training process, MobileNet and the proposed CNN obtained the best accuracy, sequentially followed by VGG16, ResNet50, and InceptionV3. The accuracy of these models is illustrated in Fig. 8b. The proposed CNN outperformed both InceptionV3 and ResNet50 and obtained a nearly similar performance as VGG16.

B. Testing Accuracy: MobileNet and ResNet50 obtained the best testing accuracy as shown in Fig. 8a. The proposed model outperformed InceptionV3 and demonstrated nearly the same performance as MobileNet and ResNet50.

C. Specificity: The proposed model obtained a similar specificity as MobileNet, ResNet50, and VGG16 and outperformed InceptionV3 as shown in Fig. 8c.

D. Sensitivity: The proposed model obtained a comparable and nearly similar sensitivity as MobileNet and ResNet50.

\section{Conclusion and Recommendations for Future Research}

New hikes in the number of COVID-19 cases are being reported all over the world. One profound symptom of COVID-19 is a lung infection. Therefore, medical imaging techniques, such as CT, can be used for COVID-19 diagnosis. This article proposed an optimized computational model for the early detection and management of COVID-19. In this model, BBO was used for selecting the layers of the deep learning model to achieve optimal accuracy. Classification accuracy was considered in the fitness function formation to enhance the prediction capability of the algorithm. The proposed model accepts images, such as CT, X-rays, positron emission tomography, lung ultrasounds, and magnetic resonance imaging, as inputs. The proposed deep learning model CNN was also compared with existing models, namely, VGG16, InceptionV3, ResNet50, and MobileNet. Results show that this model produces significant results with a training accuracy higher than those of InceptionV3 and ResNet50. This model also outperformed InceptionV3 on the test set.

Future research may adopt other optimized algorithms, such as general adversarial nets, for data augmentation to produce more significant results. Given that the dataset used in this study was limited, future studies should utilize additional datasets to further enhance the findings of this work.

Funding Statement: The authors received no specific funding for this study.

Conflicts of Interest: The authors declare that they have no conflicts of interest to report regarding the present study .

\section{References}

[1] J. Chan, F. Woo, S. Yuan, K. H. Kok, K. Kai-Wang et al., "A familial cluster of pneumonia associated with the 2019 novel coronavirus indicating person-to-person transmission: A study of a family cluster," Lancet, vol. 395, no. 10223, pp. 514-523, 2020.

[2] W. H. Organization, "Who director-general's opening remarks at the media briefing on covid-1911 march 2020," 2020. [Online]. Available: https://www.who.int/director-general/speeches/detail/whodirector-general-s-opening-remarks-at-the-media-briefing-on-covid-19.

[3] C. Lindmeier, "Statement on the second meeting of the international health regulations. Emergency committee regarding the outbreak of novel coronavirus," (2019-ncov), 2005. [Online]. Available: https://www.who.int/news-room/detail/30-01-2020-statement-onthesecond-meeting-of-the-internationalhealth-regulations, 2020. 
[4] C. Sohrabi, Z. Alsafi, N. O’Neill, M. Khan, A. Kerwan et al., "World health organization declares global emergency: A review of the 2019 novel coronavirus (COVID-19)," International Journal of Surgery, vol. 76, pp. 71-76, 2020.

[5] Q. Li, X. Guan, P. Wu, X. Wang, L. Zhou, Y. Tong et al., "Early transmission dynamics in wuhan, China, of novel coronavirus-infected pneumonia," New England Journal of Medicine, vol. 382, no. 13, pp. 1199-1207, 2020.

[6] E. Mahase, "Coronavirus: Covid-19 has killed more people than SARS and MERS combined, despite lower case fatality rate," vol. 368, pp. 843-848, 2020.

[7] C. Calisher, D. Carroll, R. Colwell, R. B. Corley, P. Daszak et al., "Statement in support of the scientists, public health professionals, and medical professionals of China combatting COVID-19," Lancet, vol. 395, no. 10226, pp. 42-43, 2020.

[8] W. Carlos, S. D. Cruz, B. Cao, S. Pasnick and S. Jamil, "COVID-19 disease due to SARS-coV-2 (novel coronavirus)," American Journal of Respiratory and Critical Care Medicine, vol. 201, no. 4, pp. 7-8, 2020.

[9] C. Huang, Y. Wang, X. Li, L. Ren, J. Zhao et al. "Clinical features of patients infected with 2019 novel coronavirus in wuhan, China," Lancet, vol. 395, no. 10223, pp. 497-506, 2020.

[10] W. Guan, Z. Ni, Y. Hu, W. Liang, C. Ou J. et al., "Clinical characteristics of coronavirus disease 2019 in China," New England Journal of Medicine, vol. 382, no. 18, pp. 1708-1720, 2020.

[11] M. Hope, D. Constantine, A. Raptis, and T. S. Henry, "Chest computed tomography for detection of coronavirus disease 2019 (COVID-19): Don't rush the science," Ann International Medicine, vol. 173, no. 2, pp. 147-148, 2020.

[12] N. Chen, M. Zhou, X. Dong, J. Qu, F. Gong et al., "Epidemiological and clinical characteristics of 99 cases of 2019 novel coronavirus pneumonia in wuhan, China: A descriptive study," Lancet, vol. 395, no. 10223 , pp. 507-513, 2020.

[13] D. Kermany, S. M. Goldbaum, H. Liang, S. L. Baxter, A. McKeown et al., "Identifying medical diagnoses and treatable diseases by image-based deep learning," Cell, vol. 172, no. 5, pp. 1122-1131, 2018.

[14] A. Alimadadi, S. Aryal, I. Manandhar, P. B. Munroe, B. Joe et al., "Artificial intelligence and machine learning to fight COVID-19," Physiological Genomics, vol. 52, no. 4, pp. 200-202, 2020.

[15] P. Hamet and J. Tremblay, "Artificial intelligence in medicine," Metabolism, vol. 69, pp. S36-S40, 2020.

[16] M. Kaur, H. K. Gianey, D. Singh and M. Sabharwal, "Multi-objective differential evolution based random forest for e-health applications," Modern Physics Letters, vol. B33, no. 5, pp. 1950-022, 2019.

[17] C. Jin, W. Chen, Y. Cao, Z. Xu, Z. Tan et al., "Development and evaluation of an artificial intelligence system for COVID-19 diagnosis," Nature Communications, vol. 11, no. 1, pp. 1-14, 2020.

[18] C. Heid, A. J. Stevens, K. J. Livak and P. M. Williams, "Real time quantitative PCR," Genome Research, vol. 6, no. 10, pp. 986-994, 1996.

[19] Y. Li and L. Xia, "Coronavirus disease 2019 (COVID-19): Role of chest CT in diagnosis and management," American Journal of Roentgenology, vol. 214, no. 6, pp. 1280-1286, 2020.

[20] J. Kanne, "Chest CT findings in 2019 novel coronavirus (2019-nCoV) infections from wuhan, China: Key points for the radiologist," Radiology, vol. 295, no. 1, pp. 16-17, 2020.

[21] Y. Fang, H. Zhang, J. Xie, M. Lin, L. Ying et al., "Sensitivity of chest CT for COVID-19: Comparison to RT-pCR.” Radiology, vol. 296, no. 2, pp. E115-E117, 2020.

[22] T. Liang, Handbook of COVID-19 Prevention and Treatment: Compiled According to Clinical Experience. Zhejiang University School of Medicine, 2020. [Online]. Available: https://smartnet.niua.org/sites/ default/files/resources/handbook_of_covid_19_prevention_en_mobile.pdf.

[23] N. Ali, C. Kaya and Z. Pamuk, "Automatic detection of coronavirus disease (covid-19) using X-ray images and deep convolutional neural networks," 2020, [Online]. Available: https://arxiv.org/abs/2003. 10849.

[24] T. A. Mpesiana, "Covid-19: Automatic detection from x-ray images utilizing transfer learning with convolutional neural networks," Physical and Engineering Sciences in Medicine, vol. 43, no. 2, pp. 635640, 2020. 
[25] A. Bernheim, X. M. M. Huang, Y. Yang and Z. A. Fayad, "Chest CT findings in coronavirus disease19 (COVID-19): Relationship to duration of infection," Radiology, vol. 295, no. 3, pp. 685-691, 2020.

[26] J. Kanne, P. Brent, P. Little, J. H. Chung, B. M. Elicker et al., "Essentials for radiologists on COVID-19: An update-Radiology scientific expert panel," vol. 296, no. 2, pp. E113-E114, 2020.

[27] J. Lei, J. Li, X. Li and X. Qi, "CT imaging of the 2019 novel coronavirus (2019-nCoV) pneumonia," Radiology, vol. 295, no. 1, pp. 18-28, 2020.

[28] D. Kermany, S. M. Goldbaum, W. Cai, H. L. Sally, L. Baxter et al., "Identifying medical diagnoses and treatable diseases by image-based deep learning," Cell, vol. 172, no. 5, pp. 1122-1131, 2018.

[29] J. Kanne, "Chest CT findings in 2019 novel coronavirus (2019-nCoV) infections from wuhan, China: Key points for the radiologist,", Radiology, vol. 295, no. 1, pp. 16-17, 2020.

[30] X. Ouyang, J. Huo, L. Xia, F. Shan, J. Liu et al., "Dual-sampling attention network for diagnosis of COVID-19 from community acquired pneumonia," IEEE Transactions on Medical Imaging, vol. 39, no. 8, pp. 2595-2605, 2020.

[31] P. Nardelli, D. J. Carretero, D. B. Pelaez, G. R. Washko, F. N. Rahaghi et al., "Pulmonary artery-vein classification in CT images using deep learning," IEEE Transactions on Medical Imaging, vol. 37, no. 11, pp. 2428-2440, 2018.

[32] S. Gerard, E. Taylor, J. Patton, G. E. Christensen, J. E. Bayouth et al., "Fissurenet: A deep learning approach for pulmonary fissure detection in CT images," IEEE Transactions on Medical Imaging, vol. 38, no. 1, pp. 156-166, 2018.

[33] A. Pezeshk, S. Hamidian, N. Petrick and B. Sahiner, "3-D convolutional neural networks for automatic detection of pulmonary nodules in chest CT," IEEE Journal of Biomedical and Health Informatics, vol. 23, no. 5, pp. 2080-2090, 2018.

[34] X. Gao, C. J. Reynolds and E. Currie, "Analysis of tuberculosis severity levels from CT pulmonary images based on enhanced residual deep learning architecture," Neurocomputing, vol. 392, pp. 233-244, 2020.

[35] Y. Pathak, P. K. Shukla, A. Tiwari, S. Stalin and S. Singh, "Deep transfer learning-based classification model for COVID-19 disease," Irbm, 2020 (In Press).

[36] E. Hemdan, M. A. Shouman and M. EsmailKarar, "Covidx-net: A framework of deep learning classifiers to diagnose covid-19 in X-ray images," 2020. arXiv preprint arXiv: 2003.11055.

[37] A. Bhandary, G. AnanthPrabhu, V. Rajinikanth, K. PalaniThanaraj, S. C. Satapathy et al., "Deeplearning framework to detect lung abnormality-A study with chest X-ray and lung CT scan images," Pattern Recognition Letters, vol. 129, pp. 271-278, 2020.

[38] S. Rajaraman, J. Siegelman, P. O. Alderson, L. S. Folio and L. R. Folio, "Iteratively pruned deep learning ensembles for covid-19 detection in chest X-rays," IEEE Access, vol. 8, pp. 115041-115050, 2020.

[39] M. Abdel-Basset, R. Mohamed, M. Elhoseny, R. K. Chakrabortty and M. Ryan, "A hybrid COVID-19 detection model using an improved marine predators algorithm and a ranking-based diversity reduction strategy," IEEE Access, vol. 8, pp. 79521-79540, 2020.

[40] A. Narini, C. Kaya and Z. Pamuk, "Automatic detection of coronavirus disease (covid-19) using x-ray images and deep convolutional neural networks," 2020. arXiv preprint arXiv: 2003.10849.

[41] D. Simon, "Biogeography-based optimization," IEEE Transactions on Evolutionary Computation, vol. 12, no. 6, pp. 702-713, 2008. 